\title{
Dini continuity of the first derivatives of generalized solutions to the Dirichlet problem for linear elliptic second order equations in nonsmooth domains
}

\author{
by Michail Borsuk (Olsztyn)
}

\begin{abstract}
We consider generalized solutions to the Dirichlet problem for linear elliptic second order equations in a domain bounded by a Dini-Lyapunov surface and containing a conical point. For such solutions we derive Dini estimates for the first order generalized derivatives.
\end{abstract}

1. Introduction. We consider generalized solutions to the Dirichlet problem for a linear uniformly elliptic second order equation in divergence form

$$
\left\{\begin{array}{l}
\frac{\partial}{\partial x_{i}}\left(a^{i j}(x) u_{x_{j}}+a^{i}(x) u\right)+b^{i}(x) u_{x_{i}}+c(x) u \\
\quad=g(x)+\frac{\partial f^{j}(x)}{\partial x_{j}}, \quad x \in G \\
u(x)=\varphi(x), \quad x \in \partial G
\end{array}\right.
$$

(summation over repeated indices from 1 to $n$ is understood), where $G \subset \mathbb{R}^{n}$ is a bounded domain with boundary $\partial G$ and $\partial G$ is a Dini-Lyapunov surface containing the origin $\mathcal{O}$ as a conical point. This last means that $\partial G \backslash \mathcal{O}$ is a smooth manifold but near $\mathcal{O}$ the domain $G$ is diffeomorphic to a cone.

Hölder estimates for the first derivatives of generalized solutions to the problem (DL) are well known in the case where the leading coefficients $a^{i j}(x)$ are Hölder continuous (see e.g. [5, 8.11] for smooth domains and [1] for domains with angular points). Here we derive Dini estimates for the first derivatives of generalized solutions of the problem (DL) in a domain with a conical boundary point under minimal smoothness conditions on the leading coefficients (Dini continuity). It should be noted that interior Dini continuity

1991 Mathematics Subject Classification: 35J.

Key words and phrases: elliptic equations, nonsmooth domains, Dini continuous, smoothness of generalized solutions. 
of the first and second derivatives of generalized solutions to the problem (DL) was investigated in $[3,7]$ under the condition of Dini continuity of the first derivatives of the leading coefficients.

We introduce the following notations and definitions:

- $[l]$ : the integral part of $l$ (if $l$ is not an integer);

- $r=|x|=\left(\sum_{i=1}^{n} x_{i}^{2}\right)^{1 / 2}$;

- $G^{\prime} \Subset G: G^{\prime}$ has compact closure contained in $G$;

- mes $G$ : volume of $G$;

- $S^{n-1}$ : the unit sphere in $\mathbb{R}^{n}$;

- $B_{r}\left(x_{0}\right)$ : the open ball in $\mathbb{R}^{n}$ of radius $r$ centered at $x_{0}$;

- $\omega_{n}=2 \pi^{n / 2} /(n \Gamma(n / 2))$ : the volume of the unit ball in $\mathbb{R}^{n}$;

- $\sigma_{n}=n \omega_{n}$ : the area of the $n$-dimensional unit sphere;

- $\mathbb{R}_{+}^{n}$ : the half-space $x_{n}>0$;

- $\Sigma$ : the hyperplane $\left\{x_{n}=0\right\}$;

- $B_{r}^{+}=B_{r} \cap \mathbb{R}_{+}^{n}$, where $x_{0} \in \overline{\mathbb{R}_{+}^{n}}$;

- $(r, \omega)$ : the spherical coordinates of $x \in \mathbb{R}^{n}$ with pole $\mathcal{O}$;

- $\Omega$ : a domain in $S^{n-1}$ with smooth $(n-2)$-dimensional boundary $\partial \Omega$;

- $G_{a}^{b}=G \cap\{(r, \omega) \mid 0 \leq a<r<b, \omega \in \Omega\}$ : a layer in $\mathbb{R}^{n}$;

- $\Gamma_{a}^{b}=\partial G \cap\{(r, \omega) \mid 0 \leq a<r<b, \omega \in \partial \Omega\}$ : the lateral surface of the layer $G_{a}^{b}$;

- $D_{i} u=u_{x_{i}}=\partial u / \partial x_{i}, D_{i j} u=u_{x_{i} x_{j}}=\partial^{2} u / \partial x_{i} \partial x_{j}$;

- $\nabla u \equiv u_{x}=\left(u_{x_{1}}, \ldots, u_{x_{n}}\right)$ : the gradient of $u(x)$;

- $\mathbf{n}=\mathbf{n}(x)=\left\{\nu_{1}, \ldots, \nu_{n}\right\}$ : the unit outward normal to $\partial G$ at the point $x$;

- $d \Omega$ : the $(n-1)$-dimensional area element of the unit sphere;

- $d \sigma$ : the $(n-1)$-dimensional area element of $\partial G$;

- $\Delta$ : the Laplacian in $\mathbb{R}^{n}$;

- $\Delta_{\omega}$ : the Laplace-Beltrami operator on the unit sphere $S^{n-1}$;

- $d(x)=\operatorname{dist}(x, \partial G \backslash \mathcal{O})$

- $\Phi(x)$ : any possible extension into $G$ of a boundary function $\varphi(x)$, i.e., $\Phi(x)=\varphi(x)$ for $x \in \partial G$;

- $\mathcal{A}(t)$ : a function defined for $t \geq 0$, nonnegative, increasing, continuous at zero, with $\lim _{t \rightarrow+0} \mathcal{A}(t)=0$.

Definition 1.1. The function $\mathcal{A}$ is called Dini continuous at zero if $\int_{0}^{d} t^{-1} \mathcal{A}(t) d t<\infty$ for some $d>0$.

Definition 1.2. The function $\mathcal{A}$ is called an $\alpha$-function, $0<\alpha<1$, on $(0, d]$ if $t^{-\alpha} \mathcal{A}(t)$ is decreasing on $(0, d]$, i.e.

$$
\mathcal{A}(t) \leq t^{\alpha} \tau^{-\alpha} \mathcal{A}(\tau), \quad 0<\tau \leq t \leq d .
$$

In particular, setting $t=c \tau, c>1$, we have

$$
\mathcal{A}(c \tau) \leq c^{\alpha} \mathcal{A}(\tau), \quad 0<\tau \leq c^{-1} d .
$$


If an $\alpha$-function $\mathcal{A}$ is Dini continuous at zero, then we say that $\mathcal{A}$ is an $\alpha$-Dini function. In that case we also define the function $\mathcal{B}(t)=$ $\int_{0}^{t}(\mathcal{A}(\tau) / \tau) d \tau$. It is obvious that $\mathcal{B}$ is increasing and continuous on $[0, d]$, and $\mathcal{B}(0)=0$. We integrate the inequality (1.1) over $\tau$ from 0 to $t$ :

$$
\mathcal{A}(t) \leq \alpha \mathcal{B}(t) \text {. }
$$

Similarly from (1.1) we derive

$$
\int_{\delta}^{d}\left(\mathcal{A}(t) / t^{2}\right) d t=\int_{\delta}^{d} t^{\alpha-2}\left(\mathcal{A}(t) / t^{\alpha}\right) d t \leq \delta^{-\alpha} \mathcal{A}(\delta) \int_{\delta}^{d} t^{\alpha-2} d t \leq(1-\alpha)^{-1} \mathcal{A}(\delta) / \delta,
$$

whence by (1.3),

$$
\begin{aligned}
\delta \int_{\delta}^{d}\left(\mathcal{A}(t) / t^{2}\right) d t & \leq(1-\alpha)^{-1} \mathcal{A}(\delta) \\
& \leq \alpha(1-\alpha)^{-1} \mathcal{B}(\delta), \quad \forall \alpha \in(0,1), 0<\delta<d .
\end{aligned}
$$

Definition 1.3. The function $\mathcal{B}$ is called equivalent to $\mathcal{A}$, written $\mathcal{A} \sim \mathcal{B}$, if there exist positive constants $C_{1}$ and $C_{2}$ such that

$$
C_{1} \mathcal{A}(t) \leq \mathcal{B}(t) \leq C_{2} \mathcal{A}(t) \quad \text { for all } t \geq 0
$$

An equivalence test is known [4, theorem of Sec. 1]: $\mathcal{A} \sim \mathcal{B}$ if and only if

$$
\varliminf_{t \rightarrow 0} \mathcal{A}(2 t) / \mathcal{A}(t)>1 \text {. }
$$

In some cases we shall consider functions $\mathcal{A}$ such that also

$$
\sup _{0<\tau \leq 1} \mathcal{A}(\tau t) / \mathcal{A}(\tau) \leq c \mathcal{A}(t), \quad \forall t \in(0, d],
$$

with some constant $c$ independent of $t$. Examples of $\alpha$-Dini functions $\mathcal{A}$ which satisfy (1.5), (1.6) with $c=1$ are:

$$
\begin{gathered}
t^{\alpha}, \quad 0 \leq t<\infty \\
t^{\alpha} \ln (1 / t), \quad t \in(0, d], d=\min \left(e^{-e}, e^{-1 / \alpha}\right), e^{-1}<\alpha<1 .
\end{gathered}
$$

We will consider the following function spaces:

- $C^{l}(\bar{G})$ : the Banach space of functions having all the derivatives of order at most $l$ (if $l=$ integer $\geq 0$ ) and of order $[l]$ (if $l$ is noninteger) continuous in $\bar{G}$ and whose $[l]$ th order partial derivatives are uniformly Hölder continuous with exponent $l-[l]$ in $\bar{G} ;|u|_{l ; G}$ is the norm of the element $u \in C^{l}(\bar{G})$; if $l \neq[l]$ then

$$
|u|_{l ; G}=\sum_{j=0}^{[l]} \sup _{G}\left|D^{j} u\right|+\sup _{|\alpha|=[l]} \sup _{\substack{x, y \in G \\ x \neq y}} \frac{\left|D^{\alpha} u(x)-D^{\alpha} u(y)\right|}{|x-y|^{l-[l]}} .
$$

- $C_{0}^{k}(G)$ : the set of functions in $C^{k}(G)$ with compact support in $G$. 
- $C^{0, \mathcal{A}}(G)$ : the set of bounded and continuous functions $f$ on $G$ with

$$
[f]_{\mathcal{A} ; G}=\sup _{\substack{x, y \in G \\ x \neq y}} \frac{|f(x)-f(y)|}{\mathcal{A}(|x-y|)}<\infty ;
$$

equipped with the norm

$$
\|f\|_{0, \mathcal{A} ; G}=|f|_{0 ; G}+[f]_{\mathcal{A} ; G},
$$

this set is a Banach space. We also define the quantity

$$
[f]_{\mathcal{A}, x}=\sup _{y \in G \backslash\{x\}} \frac{|f(x)-f(y)|}{\mathcal{A}(|x-y|)} .
$$

It is not difficult to see that if $\mathcal{A} \sim \mathcal{B}$ then $[f]_{\mathcal{A}} \sim[f]_{\mathcal{B}}$.

If $k \geq 1$ is an integer then we denote by $C^{k, \mathcal{A}}(G)$ the subspace of $C^{k}(G)$ consisting of functions whose $(k-1)$ th order partial derivatives are uniformly Lipschitz continuous and each $k$ th order derivative belongs to $C^{0, \mathcal{A}}(G)$; it is a Banach space with the norm

$$
\|f\|_{k, \mathcal{A} ; G}=|f|_{k ; G}+\sum_{|\beta|=k}\left[D^{\beta} f\right]_{\mathcal{A} ; G} .
$$

The interpolation inequality (see [8, (10.1)]) will be needed: if the domain has a Lipschitz boundary, then for any $\varepsilon>0$ there exists a constant $c(\varepsilon, G)$ such that for every $f \in C^{1, \mathcal{A}}(G)$,

$$
\sum_{i=1}^{n}\left|D_{i} f\right|_{0 ; G} \leq \varepsilon \sum_{i=1}^{n}\left[D_{i} f\right]_{\mathcal{A} ; G}+c(\varepsilon, G)|f|_{0 ; G} .
$$

- $L_{p}(G)$ : the Banach space of $p$-integrable functions $u$ on $G(p \geq 1)$ with norm $|u|_{p ; G}$.

Moreover, $\lambda=\lambda(\Omega)$ will stand for the smallest positive eigenvalue of the problem

$$
\left\{\begin{array}{l}
\Delta_{\omega} \psi+\lambda(\lambda+n-2) \psi=0, \quad \omega \in \Omega \subset S^{n-1}, \\
\psi(\omega)=0, \quad \omega \in \partial \Omega
\end{array}\right.
$$

and $c(.$.$) will be different constants depending only on the quantities ap-$ pearing in parentheses.

Let $T \subset \partial G$ be a nonempty set. Following [5, Sec. 6.2] and [8, Sec. 3] we shall say that the boundary portion $T$ is of class $C^{1, \mathcal{A}}$ if for each point $x_{0} \in T$ there are a ball $B=B\left(x_{0}\right)$, a one-to-one mapping $\psi$ of $B$ onto a ball $B^{\prime}$ and a constant $K>0$ such that:

(i) $B \cap \partial G \subset T, \psi(B \cap G) \subset \mathbb{R}_{+}^{n}$;

(ii) $\psi(B \cap \partial G) \subset \Sigma$;

(iii) $\psi \in C^{1, \mathcal{A}}(B), \psi^{-1} \in C^{1, \mathcal{A}}\left(B^{\prime}\right)$; 
(iv) $\|\psi\|_{1, \mathcal{A} ; B} \leq K,\left\|\psi^{-1}\right\|_{1, \mathcal{A} ; B^{\prime}} \leq K$.

It is not difficult to see that for $y=\psi(x)$ one has

$$
K^{-1}\left|y-y^{\prime}\right| \leq\left|x-x^{\prime}\right| \leq K\left|y-y^{\prime}\right|, \quad \forall x, x^{\prime} \in B .
$$

Lemma [8, Sec. 7, (iv)]. Let $\mathcal{A}$ be an $\alpha$-function and $f \in C^{0, \mathcal{A}}(B)$, $\psi^{-1} \in C^{1, \mathcal{A}}\left(B^{\prime}\right)$. Then $f \circ \psi^{-1} \in C^{1, \mathcal{A}}(B)$ and

$$
\left[f \circ \psi^{-1}\right]_{\mathcal{A} ; B} \leq K^{\alpha}[f]_{\mathcal{A} ; B} .
$$

2. Dini estimates of the first derivatives for the generalized Newtonian potential (cf. [5, Ch. 4]). We shall consider the Dirichlet problem for the Poisson equation

$$
\left\{\begin{array}{l}
\Delta v=\mathcal{G}+\sum_{j=1}^{n} D_{j} \mathcal{F}^{j}, \quad x \in G, \\
v(x)=0, \quad x \in \partial G .
\end{array}\right.
$$

Let $\Gamma(x-y)$ be the normalized fundamental solution of Laplace's equation. The following estimates are known (see e.g. [5, (2.12), (2.14)]):

$$
\begin{aligned}
|\Gamma(x-y)| & =|x-y|^{2-n} /\left(n(n-2) \omega_{n}\right), \quad n \geq 3, \\
\left|D_{i} \Gamma(x-y)\right| & \leq|x-y|^{1-n} /\left(n \omega_{n}\right), \\
\left|D_{i j} \Gamma(x-y)\right| & \leq|x-y|^{-n} / \omega_{n}, \\
\left|D^{\beta} \Gamma(x-y)\right| & \leq C(n, \beta)|x-y|^{2-n-|\beta|} .
\end{aligned}
$$

We define the functions

$$
z(x)=\int_{G} \Gamma(x-y) \mathcal{G}(y) d y, \quad w(x)=D_{j} \int_{G} \Gamma(x-y) \mathcal{F}^{j}(y) d y,
$$

assuming that the functions $\mathcal{G}(x)$ and $\mathcal{F}^{j}(x), j=1, \ldots, n$, are integrable on $G$. The function $z$ is called the Newtonian potential with density function $\mathcal{G}$, and $w$ is called the generalized Newtonian potential with density function $\operatorname{div} \mathcal{F}$. We now give estimates for these potentials.

Let $B_{1}=B_{R}\left(x_{0}\right), B_{2}=B_{2 R}\left(x_{0}\right)$ be concentric balls in $\mathbb{R}^{n}$ and $z, w$ be Newtonian potentials in $B_{2}$.

Lemma 1. Suppose $\mathcal{G} \in L_{p}\left(B_{2}\right), p>n / 2$, and $\mathcal{F}^{j} \in L_{\infty}\left(B_{2}\right), j=$ $1, \ldots, n$. Then

$$
\begin{aligned}
& |z|_{0 ; B_{1}} \leq c(p) R^{2 / p^{\prime}} \ln ^{1 / p^{\prime}}(1 /(2 R))|\mathcal{G}|_{p ; B_{2}}, \quad n=2, \\
& |z|_{0 ; B_{1}} \leq c(p, n) R^{2-n+n / p^{\prime}}|\mathcal{G}|_{p ; B_{2}}, \quad n \geq 3,
\end{aligned}
$$




$$
|w|_{0 ; B_{1}} \leq 2 R \sum_{j=1}^{n}\left|\mathcal{F}^{j}\right|_{0 ; B_{2}},
$$

where $1 / p+1 / p^{\prime}=1$.

P r o of. The estimates follow from inequalities (2.1), Hölder's inequality and [5, Lemma 4.1].

In the following the $D$ operator is always taken with respect to the $x$ variable.

Lemma 2 [5, Lemmas 4.1, 4.2]. Let $\partial G \in C^{1, \mathcal{A}}, \mathcal{G} \in L_{p}(G), p>n$, $\mathcal{F}^{j} \in C^{0, \mathcal{A}}(G)$, where $\mathcal{A}$ is an $\alpha$-function Dini continuous at zero. Then for any $x \in G$,

$$
\begin{aligned}
D_{i} z(x)= & \int_{G} D_{i} \Gamma(x-y) \mathcal{G}(y) d y, \\
D_{i} w(x)= & \int_{G_{0}} D_{i j} \Gamma(x-y)\left(\mathcal{F}^{j}(y)-\mathcal{F}^{j}(x)\right) d y \\
& -\mathcal{F}^{j}(x) \int_{\partial G_{0}} D_{i} \Gamma(x-y) \nu_{j} d_{y} \sigma
\end{aligned}
$$

$(i=1, \ldots, n)$; here $G_{0}$ is any domain containing $G$ for which the Gauss divergence theorem holds and $\mathcal{F}^{j}$ are extended to vanish outside $G$.

Lemma 3 (cf. [5, Lemma 4.4]). Let $\mathcal{G} \in L_{p}\left(B_{2}\right), p>n, \mathcal{F}^{j} \in C^{0, \mathcal{A}}\left(\bar{B}_{2}\right)$, where $\mathcal{A}$ is an $\alpha$-function Dini continuous at zero. Then $z, w \in C^{1, \mathcal{B}}\left(\bar{B}_{1}\right)$ and

$$
\begin{aligned}
\|z\|_{1, \mathcal{B} ; B_{1}} & \leq c\left(n, p, R, \mathcal{A}^{-1}(2 R)\right)|\mathcal{G}|_{p ; B_{2}}, \\
\|w\|_{1, \mathcal{B} ; B_{1}} & \leq c\left(n, p, \alpha, R, \mathcal{A}^{-1}(2 R), \mathcal{B}(2 R)\right) \sum_{j=1}^{n}\left\|\mathcal{F}^{j}\right\|_{0, \mathcal{A} ; B_{2}} .
\end{aligned}
$$

Pr o o f. Let $x, \bar{x} \in B_{1}$ and $G=B_{2}$. By formulas (2.5), (2.6), taking into account (2.1) and Hölder's inequality and setting $|x-y|=t, y-x=t \omega$, $d y=t^{n-1} d t d \Omega$, we have

$$
\begin{aligned}
\left|D_{i} z\right| & \leq\left(n \omega_{n}\right)^{-1} \int_{B_{2}}|x-y|^{1-n}|\mathcal{G}(y)| d y \\
& \leq\left(n \omega_{n}\right)^{-1}|\mathcal{G}|_{p ; B_{2}}\left\{\int_{B_{2}}|x-y|^{(1-n) p^{\prime}} d y\right\}^{1 / p^{\prime}} \\
& =\frac{p-1}{p-n}(2 R)^{(p-n) /(p-1)}|\mathcal{G}|_{p ; B_{2}}, \\
\left|D_{i} w(x)\right| & \leq\left(n \omega_{n}\right)^{-1} R^{1-n} \sum_{j=1}^{n}\left|\mathcal{F}^{j}(x)\right| \int_{\partial B_{2}} d_{y} \sigma
\end{aligned}
$$




$$
\begin{aligned}
& +\omega_{n}^{-1} \sum_{j=1}^{n}\left[\mathcal{F}^{j}\right]_{\mathcal{A}, x} \int_{B_{2}} \frac{\mathcal{A}(x-y)}{|x-y|^{n}} d y \\
\leq & 2^{n-1} \sum_{j=1}^{n}\left|\mathcal{F}^{j}(x)\right|+n \sum_{j=1}^{n}\left[\mathcal{F}^{j}\right]_{\mathcal{A}, x} \int_{0}^{2 R} \frac{\mathcal{A}(t)}{t} d t \\
\leq & c(n) \mathcal{B}(2 R) \sum_{j=1}^{n}\left(\left|\mathcal{F}^{j}(x)\right|+\left[\mathcal{F}^{j}\right]_{\mathcal{A}, x}\right) .
\end{aligned}
$$

Taking into account (2.5) we obtain by subtraction

$$
\left|D_{i} z(x)-D_{i} z(\bar{x})\right| \leq \int_{B_{2}}\left|D_{i} \Gamma(x-y)-D_{i} \Gamma(\bar{x}-y)\right| \cdot|\mathcal{G}(y)| d y .
$$

We set $\delta=|x-\bar{x}|, \xi=\frac{1}{2}(x-\bar{x})$ and write $B_{2}=B_{\delta}(\xi) \cup\left\{B_{2} \backslash B_{\delta}(\xi)\right\}$. Then

$$
\begin{aligned}
& \int_{B_{\delta}(\xi)}\left|D_{i} \Gamma(x-y)-D_{i} \Gamma(\bar{x}-y)\right| \cdot|\mathcal{G}(y)| d y \\
& \leq \int_{B_{\delta}(\xi)}\left|D_{i} \Gamma(x-y)\right| \cdot|\mathcal{G}(y)| d y+\int_{B_{\delta}(\xi)}\left|D_{i} \Gamma(\bar{x}-y)\right| \cdot|\mathcal{G}(y)| d y \\
& \leq\left(n \omega_{n}\right)^{-1}\left\{\int_{B_{\delta}(\xi)}|x-y|^{1-n}|\mathcal{G}(y)| d y+\int_{B_{\delta}(\xi)}|\bar{x}-y|^{1-n}|\mathcal{G}(y)| d y\right\} \\
& \leq 2\left(n \omega_{n}\right)^{-1} \int_{B_{3 \delta / 2}(x)}|x-y|^{1-n}|\mathcal{G}(y)| d y \\
& \leq 2\left(n \omega_{n}\right)^{-1}|\mathcal{G}|_{p ; B_{2}}\left(\int_{B_{3 \delta / 2}(x)}|x-y|^{(1-n) p^{\prime}} d y\right)^{1 / p^{\prime}} \\
& \leq 2\left(n \omega_{n}\right)^{-1 / p}|\mathcal{G}|_{p ; B_{2}}\left(\frac{3 \delta}{2}\right)^{1-n / p}\left\{n+(1-n) p^{\prime}\right\}^{-1 / p^{\prime}} \\
& \leq \frac{2\left(n \omega_{n}\right)^{-1 / p}(2 R)^{1-n / p}}{\left\{n+(1-n) p^{\prime}\right\}^{-1 / p^{\prime}}} \cdot \frac{\mathcal{A}(|\bar{x}-x|)}{\mathcal{A}(2 R)}|\mathcal{G}|_{p ; B_{2}}, \quad 1 / p+1 / p^{\prime}=1
\end{aligned}
$$

(here we take into account that $\delta^{\alpha} \leq(2 R)^{\alpha} \mathcal{A}(\delta) / \mathcal{A}(2 R)$ for all $\alpha>0$ by (1.1), since $\delta \leq 2 R)$. Similarly,

$$
\begin{aligned}
\int_{B_{2} \backslash B_{\delta}(\xi)}\left|D_{i} \Gamma(x-y)-D_{i} \Gamma(\bar{x}-y)\right| \cdot|\mathcal{G}(y)| d y \\
\leq|x-\bar{x}| \int_{B_{2} \backslash B_{\delta}(\xi)}\left|D D_{i} \Gamma(\widetilde{x}-y)\right| \cdot|\mathcal{G}(y)| d y
\end{aligned}
$$

(for some $\widetilde{x}$ between $x$ and $\bar{x}$ ) 


$$
\begin{aligned}
& \leq \delta \omega_{n}^{-1} \int_{|y-\xi| \geq \delta}|y-\widetilde{x}|^{-n}|\mathcal{G}(y)| d y \\
& \left.\leq 2^{n} \delta \omega_{n}^{-1} \int_{|y-\xi| \geq \delta}|y-\xi|^{-n}|\mathcal{G}(y)| d y \quad \text { (since }|y-\xi| \leq 2|y-\widetilde{x}|\right) \\
& \leq 2^{n} \delta \omega_{n}^{-1}|\mathcal{G}|_{p ; B_{2}}\left(\int_{|y-\xi| \geq \delta}|y-\xi|^{-n p^{\prime}} d y\right)^{1 / p^{\prime}} \\
& \leq 2^{n} \omega_{n}^{-1 / p}(p-1)^{1 / p^{\prime}} \delta^{1-n / p}|\mathcal{G}|_{p ; B_{2}} \\
& \leq 2^{n} \omega_{n}^{-1 / p}(p-1)^{1 / p^{\prime}}(2 R)^{1-n / p} \frac{\mathcal{A}(|x-\bar{x}|)}{\mathcal{A}(2 R)}|\mathcal{G}|_{p ; B_{2}} .
\end{aligned}
$$

From (2.11) and (2.12), taking into account (1.3), we obtain

$$
\begin{aligned}
\mid D_{i} z(x)- & D_{i} z(\bar{x}) \mid \\
& \leq c(n, p, R) \mathcal{A}^{-1}(2 R)|\mathcal{G}|_{p ; B_{2}} \mathcal{A}(|x-\bar{x}|) \\
& \leq c(n, p, R) \mathcal{A}^{-1}(2 R)|\mathcal{G}|_{p ; B_{2}} \mathcal{B}(|x-\bar{x}|), \quad \forall x, \bar{x} \in B_{1} .
\end{aligned}
$$

The first of the required estimates, (2.7), follows from (2.3) and (2.13). Now we derive the estimate (2.8).

By (2.6) for all $x, \bar{x} \in B_{1}$ we have

$$
\begin{aligned}
D_{i} w(\bar{x})-D_{i} w(x) & \\
= & \sum_{j=1}^{n}\left(\mathcal{F}^{j}(x) \mathcal{J}_{1 j}+\left(\mathcal{F}^{j}(x)-\mathcal{F}^{j}(\bar{x})\right) \mathcal{J}_{2 j}\right)+\mathcal{J}_{3} \\
& +\mathcal{J}_{4}+\sum_{j=1}^{n}\left(\mathcal{F}^{j}(x)-\mathcal{F}^{j}(\bar{x})\right) \mathcal{J}_{5 j}+\mathcal{J}_{6},
\end{aligned}
$$

where

$$
\begin{aligned}
\mathcal{J}_{1 j} & =\int_{\partial B_{2}}\left(D_{i} \Gamma(x-y)-D_{i} \Gamma(\bar{x}-y)\right) \nu_{j}(y) d_{y} \sigma \\
\mathcal{J}_{2 j} & =\int_{\partial B_{2}} D_{i} \Gamma(\bar{x}-y) \nu_{j}(y) d_{y} \sigma \\
\mathcal{J}_{3} & =\int_{B_{\delta}(\xi)} D_{i j} \Gamma(x-y)\left(\mathcal{F}^{j}(x)-\mathcal{F}^{j}(y)\right) d y \\
\mathcal{J}_{4} & =\int_{B_{\delta}(\xi)} D_{i j} \Gamma(\bar{x}-y)\left(\mathcal{F}^{j}(y)-\mathcal{F}^{j}(\bar{x})\right) d y \\
\mathcal{J}_{5 j} & =\int_{B_{2} \backslash B_{\delta}(\xi)} D_{i j} \Gamma(x-y) d y
\end{aligned}
$$




$$
\mathcal{J}_{6}=\int_{B_{2} \backslash B_{\delta}(\xi)}\left(D_{i j} \Gamma(x-y)-D_{i j} \Gamma(\bar{x}-y)\right)\left(\mathcal{F}^{j}(\bar{x})-\mathcal{F}^{j}(y)\right) d y .
$$

(Here we set again $\delta=|x-\bar{x}|, \xi=\frac{1}{2}(x-\bar{x})$ and write $B_{2}=B_{\delta}(\xi) \cup\left\{B_{2} \backslash\right.$ $\left.\left.B_{\delta}(\xi)\right\}.\right)$

We estimate these integrals by analogy with [5, pp. 58-59]:

$$
\begin{aligned}
\left|\mathcal{J}_{1 j}\right| & \leq|x-\bar{x}| \int_{\partial B_{2}}\left|D D_{i} \Gamma(\widetilde{x}-y)\right| d_{y} \sigma \\
& \leq|x-\bar{x}| n \omega_{n}^{-1} \int_{\partial B_{2}}|\widetilde{x}-y|^{-n} d_{y} \sigma \\
& \leq n^{2} 2^{n-1}|x-\bar{x}| R^{-1} \quad\left(\text { since }|\widetilde{x}-y| \geq R \text { for } y \in \partial B_{2}\right) \\
& \leq n^{2} 2^{n-1} \mathcal{A}(|x-\bar{x}|) R^{-1} \delta / \mathcal{A}(\delta) \\
& \leq n^{2} 2^{n} \mathcal{A}(|x-\bar{x}|) / \mathcal{A}(2 R) \\
& \quad \quad(\operatorname{since} \delta=|x-\bar{x}| \leq 2 R \text { and } \delta / \mathcal{A}(\delta) \leq 2 R / \mathcal{A}(2 R) \text { by }(1.1)) \\
& \leq n^{2} 2^{n} \alpha \mathcal{B}(\delta) / \mathcal{A}(2 R) \quad(\text { by }(1.3)) .
\end{aligned}
$$

Next,

$$
\begin{aligned}
\left|\mathcal{J}_{2 j}\right| & \leq 2^{n-1}, \\
\left|\mathcal{J}_{3}\right| & \leq \omega_{n}^{-1}\left[\mathcal{F}^{j}\right]_{\mathcal{A}, x} \int_{B_{\delta}(\xi)}|x-y|^{-n} \mathcal{A}(|x-y|) d y \\
& \leq \omega_{n}^{-1}\left[\mathcal{F}^{j}\right]_{\mathcal{A}, x} \int_{B_{3 \delta / 2}(x)}|x-y|^{-n} \mathcal{A}(|x-y|) d y \\
& =n\left[\mathcal{F}^{j}\right]_{\mathcal{A}, x} \int_{0}^{3 \delta / 2} t^{-1} \mathcal{A}(t) d t \\
& \leq(3 / 2)^{\alpha} n\left[\mathcal{F}^{j}\right]_{\mathcal{A}, x} \mathcal{B}(\delta) \quad(\text { by }(1.2)) .
\end{aligned}
$$

By analogy with the estimate for $\mathcal{J}_{3}$ we obtain

$$
\left|\mathcal{J}_{4}\right| \leq(3 / 2)^{\alpha} n\left[\mathcal{F}^{j}\right]_{\mathcal{A}, \bar{x}} \mathcal{B}(\delta), \quad\left|\mathcal{J}_{5 j}\right| \leq 2^{n} \quad(\text { see }[5, \text { p. 59]), }
$$

and

$$
\left|\mathcal{J}_{6}\right| \leq|x-\bar{x}| \int_{B_{2} \backslash B_{\delta}(\xi)}\left|D D_{i j} \Gamma(\widetilde{x}-y)\right| \cdot\left|\mathcal{F}^{j}(\bar{x})-\mathcal{F}^{j}(y)\right| d y
$$

(for some $\widetilde{x}$ between $x$ and $\bar{x}$ )

$$
\leq|x-\bar{x}| c(n) \int_{|y-\xi| \geq \delta}\left|\mathcal{F}^{j}(\bar{x})-\mathcal{F}^{j}(y)\right| \cdot|\widetilde{x}-y|^{-n-1} d y
$$




$$
\begin{aligned}
& \leq c(n) \delta\left[\mathcal{F}^{j}\right]_{\mathcal{A}, \bar{x}} \int_{|y-\xi| \geq \delta} \mathcal{A}(|\bar{x}-y|)|\widetilde{x}-y|^{-n-1} d y \\
& \leq 2^{n+1} c(n) \delta\left[\mathcal{F}^{j}\right]_{\mathcal{A}, \bar{x}} \int_{|y-\xi| \geq \delta} \mathcal{A}((3 / 2)|\xi-y|)|\xi-y|^{-n-1} d y \\
& \quad(\text { since }|\bar{x}-y| \leq(3 / 2)|\xi-y| \leq 3|x-\widetilde{y}|) \\
& \leq 2^{n+1} n \omega_{n} c(n)(3 / 2)^{\alpha} \delta\left[\mathcal{F}^{j}\right]_{\mathcal{A}, \bar{x}} \int_{\delta}^{R} t^{-2} \mathcal{A}(t) d t \\
& \quad\left(\operatorname{since} \mathcal{A}((3 / 2) t) \leq(3 / 2)^{\alpha} \mathcal{A}(t) \text { by }(1.2)\right) \\
& \leq \frac{\alpha}{1-\alpha}(3 / 2)^{\alpha} n \omega_{n} 2^{n+1} c(n)\left[\mathcal{F}^{j}\right]_{\mathcal{A}, \bar{x}} \mathcal{B}(\delta) \quad(\text { by }(1.4)) .
\end{aligned}
$$

Now from (2.14) and the above estimates we obtain

$$
\begin{array}{r}
\left|D_{i} w(\bar{x})-D_{i} w(x)\right| \\
\leq c(n, \alpha) \sum_{j=1}^{n}\left(\left|\mathcal{F}^{j}(x)\right| \mathcal{A}^{-1}(2 R)+\left[\mathcal{F}^{j}\right]_{\mathcal{A}, x}+\left[\mathcal{F}^{j}\right]_{\mathcal{A}, \bar{x}}\right) \mathcal{B}(|x-\bar{x}|), \\
\forall x, \bar{x} \in B_{1} .
\end{array}
$$

Finally, from (2.10) and (2.15) it follows that $w \in C^{1, \mathcal{B}}\left(B_{1}\right)$ and the estimate (2.8) holds. Lemma 3 is proved.

THEOREM 1. Let $v$ be a generalized solution of equation (PE) in $B_{2}^{+}$ with $\mathcal{G} \in L_{n /(1-\alpha)}\left(B_{2}^{+}\right), \mathcal{F}^{j} \in C^{0, \mathcal{A}}\left(\overline{B_{2}^{+}}\right)$, where $\mathcal{A}$ is an $\alpha$-function satisfying the Dini condition at zero, and let $v=0$ on $B_{2} \cap \Sigma$. Then $v \in C^{1, \mathcal{B}}\left(\overline{B_{1}^{+}}\right)$ and

$$
\|v\|_{1, \mathcal{B} ; B_{1}^{+}} \leq c\left(|v|_{0 ; B_{2}^{+}}+|\mathcal{G}|_{n /(1-\alpha) ; B_{2}^{+}}+\sum_{j=1}^{n}\left\|\mathcal{F}^{j}\right\|_{0, \mathcal{A} ; B_{2}^{+}}\right),
$$

where $c=c\left(n, \alpha, R, \mathcal{A}^{-1}(2 R), \mathcal{B}(2 R)\right)$.

Theorem 1 follows from (2.7), (2.8), representation of solutions of (PE) by means of the fundamental solution and by the same argument as in $[5$, 4.4-4.5] (see also [5, 8.11]).

\section{Dini continuity near a smooth portion of the boundary}

Theorem 2 (cf. [5, Corollary 8.36]). Let $\mathcal{A}$ be an $\alpha$-Dini function $(0<$ $\alpha<1)$ satisfying the condition (1.5). Let $T \subset \partial G$ be of class $C^{1, \mathcal{A}}$. Let $u \in W^{1}(G)$ be a weak solution of the problem (DL) with $\varphi \in C^{1, \mathcal{A}}(\partial G)$. Suppose the coefficients of the equation in (DL) satisfy the conditions 


$$
\begin{gathered}
a^{i j}(x) \xi_{i} \xi_{j} \geq \nu|\xi|^{2}, \quad \forall x \in \bar{G}, \xi \in \mathbb{R}^{n}, \\
a^{i j}, a^{i}, f^{i} \in C^{0, \mathcal{A}}(\bar{G}) \quad(i, j=1, \ldots, n), \\
b^{i}, c \in L_{\infty}(G), \quad g \in L_{n /(1-\alpha)}(G) .
\end{gathered}
$$

Then $u \in C^{1, \mathcal{B}}(G \cup T)$ and for every $G^{\prime} \Subset G \cup T$,

$$
\begin{aligned}
\|u\|_{1, \mathcal{B} ; G^{\prime}} \leq & c\left(n, T, \nu, k, d^{\prime}\right)\left(|u|_{0 ; G}+|g|_{n /(1-\alpha) ; G}\right. \\
& \left.+\sum_{i=1}^{n}\left\|f^{i}\right\|_{0, \mathcal{A} ; G}+\|\varphi\|_{1, \mathcal{A} ; \partial G}\right)
\end{aligned}
$$

where $d^{\prime}=\operatorname{dist}\left(G^{\prime}, \partial G \backslash T\right)$ and

$$
k=\max _{i, j=1, \ldots, n}\left\{\left\|a^{i j}, a^{i}\right\|_{0, \mathcal{A} ; G},\left|b^{i}, c\right|_{0 ; G}\right\} .
$$

Proof. We use the perturbation method. We freeze the leading coefficients $a^{i j}(x)$ at $x_{0} \in G \cup T$ by setting, without loss of generality, $a^{i j}\left(x_{0}\right)=\delta_{i}^{j}$ (see [5, Lemma 6.1]), and rewrite the equation of (DL) in the form (PE) for the function $v(x)=u(x)-\varphi(x)$ with

$$
\begin{aligned}
\mathcal{G}(x)= & g(x)-b^{i}(x)\left(D_{i} v+D_{i} \varphi\right)-c(x)(v(x)+\varphi(x)), \\
\mathcal{F}^{i}(x)= & \left(a^{i j}\left(x_{0}\right)-a^{i j}(x)\right) D_{j} v-a^{i j}(x) D_{j} \varphi \\
& -a^{i}(x)(v(x)+\varphi(x))+f^{i}(x) \quad(i=1, \ldots, n) .
\end{aligned}
$$

It is not difficult to observe that the conditions on the coefficients of the equation and on $T$ are invariant under maps of class $C^{1, \mathcal{A}}$. Therefore after a preliminary rectification of $T$ by means of a diffeomorphism $\psi \in C^{1, \mathcal{A}}$ it is sufficient to prove the theorem in the case $T \subset \Sigma$. This is carried out using Theorem 1 in a standard way (see $[5$, Chs. 6,8$]$ ). In this connection we use the following estimates for the functions (3.2), (3.3):

$$
\begin{aligned}
|\mathcal{G}|_{n /(1-\alpha) ; B_{2}^{+}} \leq & |g|_{n /(1-\alpha) ; B_{2}^{+}}+k\left(\sum_{i=1}^{n}\left|D_{i} v\right|_{0 ; B_{2}^{+}}+|v|_{0 ; B_{2}^{+}}\right. \\
& \left.+\sum_{i=1}^{n}\left|D_{i} \varphi\right|_{0 ; B_{2}^{+}}+|\varphi|_{0, B_{2}^{+}}\right) \\
\leq & |g|_{n /(1-\alpha) ; B_{2}^{+}}+k\left(\varepsilon \sum_{i=1}^{n}\left[D_{i} v\right]_{\mathcal{A} ; B_{2}^{+}}\right. \\
& \left.+c_{\varepsilon}|v|_{0 ; B_{2}^{+}}+|\varphi|_{1, B_{2}^{+}}\right) \quad(\text { by }(1.7)),
\end{aligned}
$$




$$
\begin{aligned}
\sum_{j=1}^{n}\left\|\mathcal{F}^{j}\right\|_{0, \mathcal{A} ; B_{2}^{+}} \leq & n k \mathcal{A}(2 R)\|\nabla v\|_{0, \mathcal{A} ; B_{2}^{+}}+k \sum_{i=1}^{n}\left|D_{i} v\right|_{0, B_{2}^{+}} \\
& +c(k)\left(|v|_{0 ; B_{2}^{+}}+\|\varphi\|_{1, \mathcal{A} ; B_{2}^{+}}\right)+\sum_{j=1}^{n}\left\|f^{j}\right\|_{0, \mathcal{A} ; B_{2}^{+}} .
\end{aligned}
$$

Taking into account once more the inequality (1.7) and the condition (1.5) that ensures the equivalence []$_{\mathcal{A}} \sim[]_{\mathcal{B}}$, from (3.4)-(3.5) we finally obtain

$$
\begin{aligned}
|\mathcal{G}|_{n /(1-\alpha) ; B_{2}^{+}} & +\sum_{j=1}^{n}\left\|\mathcal{F}^{j}\right\|_{0, \mathcal{A} ; B_{2}^{+}} \\
\leq & k(\varepsilon+n \mathcal{A}(2 R))\|v\|_{1, \mathcal{B} ; B_{2}^{+}}+c_{\varepsilon}(k)\left(|v|_{0 ; B_{2}^{+}}+\|\varphi\|_{1, \mathcal{A} ; B_{2}^{+}}\right) \\
& +\sum_{j=1}^{n}\left\|f^{j}\right\|_{0, \mathcal{A} ; B_{2}^{+}}+|\mathbf{I}| \mathbf{|}_{n /(1-\alpha) ; B_{2}^{+}} \quad \text { for all } \varepsilon>0 .
\end{aligned}
$$

Since $\mathcal{A}$ is continuous, choosing $\varepsilon, R>0$ sufficiently small we obtain the desired assertion and the estimate (3.1) in a standard way from (2.16) and (3.6).

4. Dini continuity near the conical point. We consider the problem (DL) under the following assumptions:

(i) $\partial G$ is a Dini-Lyapunov surface with conical point $\mathcal{O}$;

(ii) the uniform ellipticity holds:

$$
\nu \xi^{2} \leq a^{i j}(x) \xi_{i} \xi_{j} \leq \mu \xi^{2}, \quad \forall x \in G, \xi \in \mathbb{R}^{n},
$$

where $\nu, \mu=$ const $>0$ and $a^{i j}(0)=\delta_{i}^{j}(i, j=1, \ldots, n)$;

(iii) $a^{i j}, a^{i} \in C^{0, \mathcal{A}}(G)(i, j=1, \ldots, n)$ where $\mathcal{A}$ is an $\alpha$-Dini function on $(0, d], \alpha \in(0,1)$, satisfying the conditions (1.5)-(1.6) and also

$$
\begin{gathered}
\sup _{0<\varrho \leq 1} \varrho^{\lambda-1} / \mathcal{A}(\varrho) \leq \text { const }, \\
|x|\left(\sum\left(b^{i}(x)\right)^{2}\right)^{1 / 2}+|x|^{2}|c(x)| \leq \mathcal{A}(|x|) ;
\end{gathered}
$$

(iv) $g \in L_{n /(1-\alpha)}(G), \varphi \in C^{1, \mathcal{A}}(\partial G), f^{j} \in C^{0, \mathcal{A}}(\bar{G}), j=1, \ldots, n$;

(v) $\int_{G} r^{4-n-2 \lambda} \mathcal{H}^{-1}(r) g^{2}(x) d x<\infty$,

$$
\int_{G} r^{2-n-2 \lambda} \mathcal{H}^{-1}(r)\left(\sum_{j=1}^{n}\left|f^{j}\right|^{2}+|\nabla \Phi|^{2}+r^{-2} \Phi^{2}\right) d x<\infty,
$$

where $\mathcal{H}$ is a continuous increasing function satisfying the Dini condition at $t=0$. 
THEOREM 3. Let $u$ be a generalized solution of (DL) and suppose that assumptions (i) $-(\mathrm{v})$ are satisfied. Then there exist $d>0$ and a constant $c>0$ independent of $u$ and depending only on parameters and norms of the given functions appearing in assumptions (i) $-(\mathrm{v})$, such that

$$
\begin{aligned}
|u(x)| \leq & c|x| \mathcal{A}(|x|)\left(|\mathbf{g}|_{n /(1-\alpha) ; G}+\sum_{i=1}^{n}\left\|f^{i}\right\|_{0, \mathcal{A} ; G}+\|\varphi\|_{1, \mathcal{A} ; \partial G}\right. \\
& +\left\{\int _ { G } \left(r^{4-n-2 \lambda} \mathcal{H}^{-1}(r) g^{2}(x)+r^{2-n-2 \lambda} \mathcal{H}^{-1}(r)\right.\right. \\
& \times \sum_{i=1}^{n}\left|f^{i}(x)\right|^{2}+r^{2-n-2 \lambda} \mathcal{H}^{-1}(r)|\nabla \Phi|^{2} \\
& \left.\left.\left.+|u|^{2}+|\nabla u|^{2}\right) d x\right\}^{1 / 2}\right), \quad \forall x \in G_{0}^{d}, \\
|\nabla u(x)| \leq & c \mathcal{A}(|x|)\left(|g|_{n /(1-\alpha) ; G}+\sum_{i=1}^{n}\left\|f^{i}\right\|_{0, \mathcal{A} ; G}+\|\varphi\|_{1, \mathcal{A} ; \partial G}\right. \\
& +\left\{\int _ { G } \left(r^{4-n-2 \lambda} \mathcal{H}^{-1}(r) g^{2}(x)+r^{2-n-2 \lambda} \mathcal{H}^{-1}(r)\right.\right. \\
& \times \sum_{i=1}^{n}\left|f^{i}(x)\right|^{2}+r^{2-n-2 \lambda} \mathcal{H}^{-1}(r)|\nabla \Phi|^{2} \\
& \left.\left.\left.+|u|^{2}+|\nabla u|^{2}\right) d x\right\}^{1 / 2}\right), \quad \forall x \in G_{0}^{d} .
\end{aligned}
$$

Proof. We use Kondrat'ev's method of layers: we move away from the conical point of $\varrho>0$ and work in $G_{\varrho / 4}^{2 \varrho}$; after the change of variables $x=\varrho x^{\prime}$ the layer $G_{\varrho / 4}^{2 \varrho}$ takes the position of a fixed domain $G_{1 / 4}^{2}$ with smooth boundary.

$1^{\circ}$. We consider a solution $u$ in the domain $G_{0}^{2 d}$ with some positive $d \ll 1$; then $u$ is a weak solution in $G_{0}^{2 d}$ of the problem

$(\mathrm{DL})_{0,2 d}\left\{\begin{aligned} \frac{\partial}{\partial x_{i}} & \left(a^{i j}(x) u_{x_{j}}+a^{i}(x) u\right)+b^{i}(x) u_{x_{i}}+c(x) u \\ = & g(x)+\frac{\partial f^{j}}{\partial x_{j}}, \quad x \in G_{0}^{2 d}, \\ u(x) & =\varphi(x), \quad x \in \Gamma_{0}^{2 d} \subset \partial G_{0}^{2 d} .\end{aligned}\right.$

We make the change of variables $x=\varrho x^{\prime}$ and set $v\left(x^{\prime}\right)=\varrho^{-1} \mathcal{A}^{-1}(\varrho) u\left(\varrho x^{\prime}\right)$, 
$\varrho \in(0, d), 0<d \ll 1$. Then $v$ satisfies in $G_{1 / 4}^{2}$ the problem

$$
\left\{\begin{array}{l}
\frac{\partial}{\partial x_{i}^{\prime}}\left(a^{i j}\left(\varrho x^{\prime}\right) v_{x_{j}^{\prime}}+\varrho a^{i}\left(\varrho x^{\prime}\right) v\right)+\varrho b^{i}\left(\varrho x^{\prime}\right) v_{x_{i}^{\prime}}+\varrho^{2} c\left(\varrho x^{\prime}\right) v \\
\quad=\mathcal{A}^{-1}(\varrho) \sum_{j=1}^{n} \frac{\partial f^{j}\left(\varrho x^{\prime}\right)}{\partial x_{j}^{\prime}}+\varrho \mathcal{A}^{-1}(\varrho) g\left(\varrho x^{\prime}\right), \quad x^{\prime} \in G_{1 / 4}^{2}, \\
v\left(x^{\prime}\right)=\varrho^{-1} \mathcal{A}^{-1}(\varrho) \varphi\left(\varrho x^{\prime}\right), \quad x^{\prime} \in \Gamma_{1 / 4}^{2} .
\end{array}\right.
$$

To solve this problem we use Theorem 2 . We check its assumptions. Since under assumption (ii), $\mathcal{A}$ is increasing, $\varrho \in(0, d)$ and $0<d \ll 1$, from the inequality $\varrho^{-1}|x-y| \geq|x-y|$ for $\varrho \in(0, d)$ it follows that

$$
\mathcal{A}\left(\left|x^{\prime}-y^{\prime}\right|\right)=\mathcal{A}\left(\varrho^{-1}|x-y|\right) \geq \mathcal{A}(|x-y|)
$$

and by (iii) we have

$$
\begin{aligned}
\sum_{i, j}\left\|a^{i j}(\varrho \cdot)\right\|_{0, \mathcal{A} ; G_{1 / 4}^{2}}+\varrho & \sum_{i}\left\|a^{i}(\varrho \cdot)\right\|_{0, \mathcal{A} ; G_{1 / 4}^{2}} \\
& \leq \sum_{i, j}\left\|a^{i j}\right\|_{0, \mathcal{A} ; G_{\varrho / 4}^{2 \varrho}}+d \sum_{i}\left\|a^{i}\right\|_{0, \mathcal{A} ; G_{\varrho / 4}^{2 \varrho}}<\infty .
\end{aligned}
$$

Further, let $\Phi$ be a regularity preserving extension of the boundary function $\varphi$ into a domain $G_{\varepsilon}^{d}$ for $\varepsilon>0$ (such an extension exists; see e.g. [5, Lemma 6.38]).

Since $\varphi \in C^{1, \mathcal{A}}(\partial G)$ we have

$$
\|\Phi\|_{1, \mathcal{A} ; G_{\varrho / 4}^{2 \varrho}} \leq c(G)\|\varphi\|_{1, \mathcal{A} ; \Gamma_{\varrho / 4}^{2 \varrho}} \leq \text { const. }
$$

By definition of the norm in $C^{1, \mathcal{A}}$ we obtain

$$
\sup _{\substack{x, y \in G_{\varrho / 4}^{2 \varrho} \\ x \neq y}} \frac{|\nabla \Phi(x)-\nabla \Phi(y)|}{\mathcal{A}(|x-y|)} \leq\|\Phi\|_{1, \mathcal{A} ; G_{\varrho / 4}^{2 \varrho}} \leq c(G)\|\varphi\|_{1, \mathcal{A} ; \Gamma_{\varrho / 4}^{2 \varrho}} .
$$

Now we show that by (v) and the smoothness of $\varphi$,

$$
|\varphi(x)| \leq c|x| \mathcal{A}(|x|), \quad|\nabla \Phi(x)| \leq c \mathcal{A}(|x|), \quad \forall x \in G_{\varrho / 4}^{2 \varrho} .
$$

Indeed, from

$$
\varphi(x)-\varphi(0)=\int_{0}^{1} \frac{d}{d \tau} \Phi(\tau x) d \tau=x_{i} \int_{0}^{1} \frac{\partial \Phi(\tau x)}{\partial\left(\tau x_{i}\right)} d \tau
$$

by Hölder's inequality we have

$$
|\varphi(x)-\varphi(0)| \leq r|\nabla \Phi| .
$$


From (iv) it follows that

$$
\begin{aligned}
& \int_{G_{0}^{\varrho}}\left(r^{2-n}|\nabla \Phi|^{2}+r^{-n}|\varphi|^{2}\right) d x \\
& \quad=\int_{G_{0}^{\varrho}}\left(r^{2-n-2 \lambda} \mathcal{H}^{-1}(r)|\nabla \Phi|^{2}+r^{-n-2 \lambda} \mathcal{H}^{-1}(r)|\varphi|^{2}\right)\left(r^{2 \lambda} \mathcal{H}(r)\right) d x \\
& \quad \leq \operatorname{const} \varrho^{2 \lambda} \mathcal{H}(\varrho) .
\end{aligned}
$$

Since $|\varphi(0)| \leq|\varphi(x)|+|\varphi(x)-\varphi(0)|$, by (4.6) we obtain

$$
|\varphi(0)| \leq|\varphi(x)|+r|\nabla \Phi| .
$$

Squaring both sides, multiplying by $r^{-n}$ and integrating over $G_{0}^{\varrho}$ we obtain

$$
\varphi^{2}(0) \int_{G_{0}^{e}} r^{-n} d x \leq 2 \int_{G_{0}^{e}}\left(r^{-n} \varphi^{2}(x)+r^{2-n}|\nabla \Phi|^{2}\right) d x<\infty
$$

by (4.7). Since

$$
\int_{G_{0}^{\varrho}} r^{-n} d x=\operatorname{mes} \Omega \int_{0}^{\varrho} \frac{d r}{r}=\infty,
$$

the assumption $\varphi(0) \neq 0$ contradicts (4.8). Thus $\varphi(0)=0$. Then from (4.4) we have

$$
\begin{aligned}
|\nabla \Phi(x)-\nabla \Phi(y)| & \leq \operatorname{const} \mathcal{A}(|x-y|)\|\varphi\|_{1, \mathcal{A} ; \Gamma_{\varrho / 4}^{2 \varrho}}, \quad \forall x, y \in G_{\varrho / 4}^{2 \varrho} \\
|\nabla \Phi(y)| & \leq|\nabla \Phi(x)-\nabla \Phi(y)|+|\nabla \Phi(x)| \\
& \leq c \mathcal{A}(|x-y|)\|\varphi\|_{1, \mathcal{A} ; \Gamma_{\varrho / 4}^{2 \varrho}}+|\nabla \Phi(x)| .
\end{aligned}
$$

Hence considering $y$ to be fixed in $G_{\varrho / 4}^{2 \varrho}$ and $x$ variable, we get

$$
\begin{aligned}
|\nabla \Phi(y)|^{2} \int_{G_{\varrho / 4}^{2 \varrho}} r^{2-n} d x \leq & 2 c^{2}\|\varphi\|_{1, \mathcal{A} ; \Gamma_{\varrho / 4}^{2 \varrho}} \int_{G_{\varrho / 4}^{2 \varrho}} r^{2-n} \mathcal{A}^{2}(|x-y|) d x \\
& +2 \int_{G_{\varrho / 4}^{2 \varrho}} r^{2-n}|\nabla \Phi(x)|^{2} d x
\end{aligned}
$$

or by $(4.7)$,

$$
\varrho^{2}|\nabla \Phi(y)|^{2} \leq c\left(\operatorname{mes} \Omega, k_{1}\right)\left(\varrho^{2} \mathcal{A}^{2}(\varrho)+\varrho^{2 \lambda} \mathcal{H}(\varrho)\right), \quad \forall y \in G_{\varrho / 4}^{2 \varrho} .
$$

Hence the assumption (4.1) yields the second inequality of (4.5). Now the first inequality of (4.5) follows from (4.6) and $\varphi(0)=0$. Thus (4.5) is proved. 
Now we obtain

$$
\begin{aligned}
& \varrho^{-1} \mathcal{A}^{-1}(\varrho)\|\varphi(\varrho \cdot)\|_{1, \mathcal{A} ; \Gamma_{1 / 4}^{2}} \\
& \leq c \varrho^{-1} \mathcal{A}^{-1}(\varrho)\|\Phi(\varrho \cdot)\|_{1, \mathcal{A} ; G_{1 / 4}^{2}} \\
&=c \varrho^{-1} \mathcal{A}^{-1}(\varrho)\left\{\sup _{x^{\prime} \in G_{1 / 4}^{2}}\left|\Phi\left(\varrho x^{\prime}\right)\right|+\sup _{x^{\prime} \in G_{1 / 4}^{2}}\left|\nabla^{\prime} \Phi\left(\varrho x^{\prime}\right)\right|\right. \\
&\left.+\sup _{x^{\prime}, y^{\prime} \in G_{1 / 4}^{2}} \frac{\left|\nabla^{\prime} \Phi\left(\varrho x^{\prime}\right)-\nabla^{\prime} \Phi\left(\varrho y^{\prime}\right)\right|}{\mathcal{A}\left(\left|x^{\prime}-y^{\prime}\right|\right)}\right\} \\
& \leq c_{1}+c \mathcal{A}^{-1}(\varrho) \sup _{x, y \in G_{\varrho / 4}^{2 \varrho}} \frac{|\nabla \Phi(x)-\nabla \Phi(y)|}{\mathcal{A}\left(\varrho^{-1}|x-y|\right)} \\
&= c_{1}+c[\nabla \Phi]_{0, \mathcal{A} ; G_{\varrho / 4}^{2 \varrho}} \mathcal{A}^{-1}(\varrho) \sup _{0<t<4 \varrho} \frac{\mathcal{A}(t)}{\mathcal{A}\left(\varrho^{-1} t\right)} \\
& \leq \text { const }, \quad \forall \varrho \in(0, d),
\end{aligned}
$$

by (4.5), since by (1.6),

$$
\sup _{0<t<4 \varrho} \frac{\mathcal{A}(t)}{\mathcal{A}\left(\varrho^{-1} t\right)}=\sup _{0<\tau<4} \frac{\mathcal{A}(\tau \varrho)}{\mathcal{A}(\tau)} \leq c \mathcal{A}(\varrho) .
$$

In the same way we have

(4.10) $\quad \mathcal{A}^{-1}(\varrho)\left\|f^{j}\right\|_{0, \mathcal{A} ; G_{1 / 4}^{2}}$

$$
=\mathcal{A}^{-1}(\varrho)\left(\left|f^{j}\right|_{0 ; G_{\varrho / 4}^{2 \varrho}}+\sup _{\substack{x, y \in G_{\varrho / 4}^{2 \varrho} \\ x \neq y}} \frac{\left|f^{j}(x)-f^{j}(y)\right|}{\mathcal{A}\left(\varrho^{-1}|x-y|\right)}\right) .
$$

Since $f^{j} \in C^{0, \mathcal{A}}(\bar{G})$, we get

(4.11) $\quad\left|f^{j}(x)-f^{j}(y)\right| \leq \widetilde{c}_{j} \mathcal{A}(|x-y|), \quad \forall x, y \in G_{\varrho / 4}^{2 \varrho}$,

$$
\begin{aligned}
\int_{G_{0}^{\varrho}} r^{2-n}\left|f^{j}(x)\right|^{2} d x & =\int_{G_{0}^{\varrho}}\left(r^{2-n-2 \lambda} \mathcal{H}^{-1}(r)\left|f^{j}(x)\right|^{2}\right)\left(\mathcal{H}(r) r^{2 \lambda}\right) d x \\
& \leq \operatorname{const} \varrho^{2 \lambda} \mathcal{H}(\varrho)
\end{aligned}
$$

by (v). Now fix $y$ in $G_{\varrho / 4}^{2 \varrho}$. Then

$$
\left|f^{j}(y)\right| \leq\left|f^{j}(x)\right|+\left|f^{j}(x)-f^{j}(y)\right| \leq\left|f^{j}(x)\right|+\widetilde{c}_{j} \mathcal{A}(|x-y|) .
$$

Hence

$$
\left|f^{j}(y)\right|^{2} \int_{G_{\varrho / 4}^{2 \varrho}} r^{2-n} d x \leq 2 \int_{G_{\varrho / 4}^{2 \varrho}} r^{2-n}\left|f^{j}(x)\right|^{2} d x+2 \widetilde{c}_{j}^{2} \int_{G_{\varrho / 4}^{2 \varrho}} r^{2-n} \mathcal{A}^{2}(|x-y|) d x .
$$


Calculations and (4.12) give

$$
\varrho^{2}\left|f^{j}(y)\right|^{2} \leq c\left(\widetilde{c}_{j}, k_{1}, \text { mes } \Omega\right)\left(\varrho^{2} \mathcal{A}^{2}(\varrho)+\varrho^{2 \lambda} \mathcal{H}(\varrho)\right), \quad \forall y \in G_{\varrho / 4}^{2 \varrho} .
$$

Hence by the assumption (4.1) it follows that

$$
\left|f^{j}(x)\right| \leq c_{j} \mathcal{A}(\varrho), \quad \forall x \in G_{\varrho / 4}^{2 \varrho}, j=1, \ldots, n .
$$

Further, in the same way as in the proof of (4.9),

$$
\begin{aligned}
\sup _{\substack{x, y \in G^{2 \varrho} \\
x \neq y}} \frac{\left|f^{j}(x)-f^{j}(y)\right|}{\mathcal{A}\left(\varrho^{-1}|x-y|\right)} & \leq\left[f^{j}\right]_{0, \mathcal{A} ; G_{\varrho / 4}^{2 \varrho}} \sup _{0<t<4 \varrho} \frac{\mathcal{A}(t)}{\mathcal{A}\left(\varrho^{-1} t\right)} \\
& \leq c \mathcal{A}(\varrho)\left[f^{j}\right]_{0, \mathcal{A} ; G_{\varrho / 4}^{2 \varrho}}
\end{aligned}
$$

Now from (4.10), (4.13) and (4.14) we obtain

$$
\mathcal{A}^{-1}(\varrho) \sum_{j=1}^{n}\left|f^{j}\right|_{0, \mathcal{A} ; G_{1 / 4}^{2}} \leq \text { const. }
$$

It remains to verify the finiteness of $\mid \varrho \mathcal{A}(\varrho)^{-1} g\left(\varrho x^{\prime}\right) \mathbf{|}_{n /(1-\alpha) ; G_{1 / 4}^{2}}$. We have

$$
\begin{aligned}
& \varrho \mathcal{A}^{-1}(\varrho)\left(\int_{G_{1 / 4}^{2}}\left|g\left(\varrho x^{\prime}\right)\right|^{n /(1-\alpha)} d x^{\prime}\right)^{(1-\alpha) / n} \\
&=\varrho^{\alpha} \mathcal{A}^{-1}(\varrho)\left(\int_{G_{\varrho / 4}^{2 \varrho}}|g(x)|^{n /(1-\alpha)} d x\right)^{(1-\alpha) / n} \\
& \leq d^{\alpha} \mathcal{A}^{-1}(d)\left(\int_{G_{\varrho / 4}^{2 \varrho}}|g(x)|^{n /(1-\alpha)} d x\right)^{(1-\alpha) / n} \\
& \leq \text { const, } \quad \forall \varrho \in(0, d),
\end{aligned}
$$

by the condition (1.1). Thus the conditions of Theorem 2 are satisfied.

By this theorem we have

$$
\begin{aligned}
& \quad\|v\|_{1, \mathcal{B} ; G_{1 / 2}^{1}} \\
& \leq c\left\{n, \nu, G, \max _{i, j=1, \ldots, n}\left(\left\|a^{i j}(\varrho \cdot)\right\|_{0, \mathcal{A} ; G_{1 / 4}^{2}}, \varrho\left\|a^{i}(\varrho \cdot)\right\|_{0, \mathcal{A} ; G_{1 / 4}^{2}}\right), \mathcal{A}(2 \varrho)\right\} \\
& \times\left(|v|_{0 ; G_{1 / 4}^{2}}+\varrho^{-1} \mathcal{A}^{-1}(\varrho)\|\varphi(\varrho \cdot)\|_{1, \mathcal{A} ; \Gamma_{1 / 4}^{2}}+\varrho \mathcal{A}^{-1}(\varrho) \mathbf{|} g(\varrho \cdot) \mathbf{|}_{n /(1-\alpha) ; G_{1 / 4}^{2}}\right. \\
& \left.+\mathcal{A}^{-1}(\varrho) \sum_{j=1}^{n}\left\|f^{j}(\varrho \cdot)\right\|_{0, \mathcal{A} ; G_{1 / 4}^{2}}\right), \quad \forall \varrho \in(0, d) .
\end{aligned}
$$


$2^{\circ}$. To estimate $|v|_{0 ; G_{1 / 4}^{2}}$ we use the local estimate at the boundary of the maximum of the modulus of a solution [5, Theorem 8.25]. We check the assumptions of that theorem. To this end, set

$$
z\left(x^{\prime}\right)=v\left(x^{\prime}\right)-\varrho^{-1} \mathcal{A}^{-1}(\varrho) \Phi\left(\varrho x^{\prime}\right)
$$

and write the problem for the function $z$ :

$$
\left\{\begin{array}{l}
\frac{\partial}{\partial x_{i}^{\prime}}\left(a^{i j}\left(\varrho x^{\prime}\right) z_{x_{j}^{\prime}}+\varrho a^{i}\left(\varrho x^{\prime}\right) z\right)+\varrho b^{i}\left(\varrho x^{\prime}\right) z_{x_{i}^{\prime}}+\varrho^{2} c\left(\varrho x^{\prime}\right) z \\
\quad=G\left(x^{\prime}\right)+\frac{\partial F^{j}\left(x^{\prime}\right)}{\partial x_{j}^{\prime}}, \quad x^{\prime} \in G_{1 / 4}^{2}, \\
z\left(x^{\prime}\right)=0, \quad x^{\prime} \in \Gamma_{1 / 4}^{2},
\end{array}\right.
$$

where

$$
\begin{aligned}
G\left(x^{\prime}\right) \equiv & \varrho \mathcal{A}^{-1}(\varrho) g\left(\varrho x^{\prime}\right)-\mathcal{A}^{-1}(\varrho) b^{i}\left(\varrho x^{\prime}\right) \Phi_{x_{i}^{\prime}}\left(\varrho x^{\prime}\right) \\
& -\varrho \mathcal{A}^{-1}(\varrho) c\left(\varrho x^{\prime}\right) \Phi\left(\varrho x^{\prime}\right) \\
F^{i}\left(x^{\prime}\right) \equiv & \mathcal{A}^{-1}(\varrho) f^{i}\left(\varrho x^{\prime}\right)-\varrho^{-1} \mathcal{A}^{-1}(\varrho) a^{i j}\left(\varrho x^{\prime}\right) \Phi_{x_{j}^{\prime}}\left(\varrho x^{\prime}\right) \\
& -\mathcal{A}^{-1}(\varrho) a^{i}\left(\varrho x^{\prime}\right) \Phi\left(\varrho x^{\prime}\right) \quad(i=1, \ldots, n) .
\end{aligned}
$$

First we verify the smoothness of the coefficients (see the remark at the end of $[5,8.10])$. Let $q>n$. We have

$$
\begin{aligned}
\int_{G_{1 / 4}^{2}}\left|\varrho a^{i}\left(\varrho x^{\prime}\right)\right|^{q} d x^{\prime} & =\varrho^{q-n} \int_{G_{\varrho / 4}^{2 \varrho}}\left|a^{i}(x)\right|^{q} d x \\
& \leq c_{2}(G) d^{q}\left\|a^{i}\right\|_{0, \mathcal{A} ; G}^{q}, \quad \forall \varrho \in(0, d) .
\end{aligned}
$$

By (iii) we also obtain

$$
\begin{aligned}
\int_{G_{1 / 4}^{2}}\left|\varrho b^{i}\left(\varrho x^{\prime}\right)\right|^{q} d x^{\prime} & =\varrho^{q-n} \int_{G_{\varrho / 4}^{2 \varrho}}\left|b^{i}(x)\right|^{q} d x \leq 4^{q} \varrho^{-n} \int_{G_{\varrho / 4}^{2 \varrho}}\left|r b^{i}(x)\right|^{q} d x \\
& \leq 4^{q} \varrho^{-n} \int_{G_{\varrho / 4}^{2 \varrho}} \mathcal{A}^{q}(r) d x \leq 2^{n+2 q} \int_{G_{\varrho / 4}^{2 \varrho}} r^{-n} \mathcal{A}^{q}(r) d x \\
& =2^{n+2 q} \operatorname{mes} \Omega \int_{\varrho / 4}^{2 \varrho} \frac{\mathcal{A}^{q}(r)}{r} d r \\
& \leq 2^{n+2 q} \operatorname{mes} \Omega \cdot \mathcal{A}^{q-1}(2 d) \int_{0}^{2 d} \frac{\mathcal{A}(r)}{r} d r,
\end{aligned}
$$

$$
\int_{G_{1 / 4}^{2}}\left|\varrho^{2} c\left(\varrho x^{\prime}\right)\right|^{q / 2} d x^{\prime}=\varrho^{q-n} \int_{G_{\varrho / 4}^{2 \varrho}}|c(x)|^{q / 2} d x
$$




$$
\begin{aligned}
& \leq 4^{q} \varrho^{-n} \int_{G_{\varrho / 4}^{2 \varrho}}\left|r^{2} c(x)\right|^{q / 2} d x \\
& \leq 2^{2 q+n} \int_{G_{\varrho / 4}^{2 \varrho}} r^{-n} \mathcal{A}^{q / 2}(r) d x \\
& \leq 2^{2 q+n} \operatorname{mes} \Omega \cdot \mathcal{A}^{(q-2) / 2}(2 d) \int_{0}^{2 d} \frac{\mathcal{A}(r)}{r} d r,
\end{aligned}
$$

for $q>n$ and all $\varrho \in(0, d)$.

In the same way from (4.17) we get

$$
\begin{aligned}
\varrho \mathcal{A}^{-1}(\varrho) \mid & G\left(x^{\prime}\right) \mathbf{I}_{q / 2 ; G_{1 / 4}^{2}} \\
= & \varrho \mathcal{A}^{-1}(\varrho)\left(\int _ { G _ { \varrho / 4 } ^ { 2 \varrho } } \varrho ^ { - n } \left\{|g(x)|^{q / 2}\right.\right. \\
& \left.\left.+\left(\sum_{i=1}^{n}\left|b^{i}(x)\right|\right)^{q / 2}|\nabla \Phi|^{q / 2}+|c(x)|^{q / 2}|\Phi(x)|^{q / 2}\right\} d x\right)^{2 / q} .
\end{aligned}
$$

By (iv) setting $q=n /(1-\alpha)>n$ and applying Hölder's inequality we obtain

$$
\begin{aligned}
\varrho \mathcal{A}^{-1}(\varrho)\left(\int_{G_{\varrho / 4}^{2 \varrho}} \varrho^{-n}|g(x)|^{q / 2} d x\right)^{2 / q} \\
\quad \leq c \varrho^{\alpha} \mathcal{A}^{-1}(\varrho)\left(\int_{G_{\varrho / 4}^{2 \varrho}} \varrho^{-n / 2}|g(x)|^{q / 2} d x\right)^{2 / q} \\
\leq c \varrho^{\alpha} \mathcal{A}^{-1}(\varrho)|g|_{q ; G_{\varrho / 4}^{2 \varrho}}(\operatorname{mes} \Omega \ln 8)^{1 / q} \\
\leq c(d, \alpha, q, \operatorname{mes} \Omega, \mathcal{A}(d)) \mid g \mathbf{|}_{q ; G_{\varrho / 4}^{2 \varrho}}
\end{aligned}
$$

since by $(1.1), \varrho^{\alpha} \mathcal{A}^{-1}(\varrho) \leq d^{\alpha} \mathcal{A}^{-1}(d)$ for all $\varrho \in(0, d)$. Similarly,

$$
\begin{aligned}
& \varrho \mathcal{A}^{-1}(\varrho)\left(\int_{G_{\varrho / 4}^{2 \varrho}} r^{-n}\right. \\
&\left.\quad \times\left\{\left(\sum_{i=1}^{n}\left|b^{i}(x)\right|\right)^{q / 2}|\nabla \Phi|^{q / 2}+|c(x)|^{q / 2}|\Phi(x)|^{q / 2}\right\} d x\right)^{2 / q} \\
& \leq c(\operatorname{mes} \Omega)^{2 / q}\|\varphi\|_{1, \mathcal{A} ; G_{\varrho / 4}^{2 \varrho}} \mathcal{A}^{(q-2) / q}(\varrho) \int_{\varrho / 4}^{2 \varrho} \frac{\mathcal{A}(r)}{r} d r .
\end{aligned}
$$


From (4.22)-(4.24) we obtain

$$
\begin{aligned}
& |G(\varrho \cdot)|_{q / 2 ; G_{1 / 4}^{2}} \\
& \leq \operatorname{const}\left(q, \alpha, d, \operatorname{mes} \Omega, \mathcal{A}(d), \int_{\varrho / 4}^{2 \varrho} \frac{\mathcal{A}(r)}{r} d r\right) \\
& \quad \times\left(|g|_{q ; G_{\varrho / 4}^{2 \varrho}}+\|\varphi\|_{1, \mathcal{A} ; \Gamma_{\varrho / 4}^{2 \varrho}}\right), \quad q=n /(1-\alpha)>n .
\end{aligned}
$$

Finally, in the same way from (4.18) we have

$$
\begin{aligned}
\sum_{i=1}^{n} \int_{G_{1 / 4}^{2}}\left|F^{i}\left(x^{\prime}\right)\right|^{q} d x^{\prime} & \\
\leq & c\left(q, G, \max _{j=1, \ldots, n}\left\{\sum_{i=1}^{n}\left\|a^{i j}\right\|_{0, \mathcal{A} ; G}^{q}, \sum_{i=1}^{n}\left\|a^{i}\right\|_{0, \mathcal{A} ; G}^{q}\right\}\right) \\
& \times \int_{G_{\varrho / 4}^{2 \varrho}} r^{-n} \mathcal{A}^{-q}(r)\left(\sum_{i=1}^{n}\left|f^{i}(x)\right|^{q}+|\nabla \Phi|^{q}+|\Phi|^{q}\right) d x .
\end{aligned}
$$

It follows from (4.5) as $\varrho \rightarrow+0$ that $|\nabla \Phi(0)|=0$. Therefore

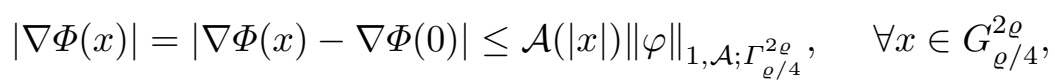

and hence

$$
|\Phi(x)| \leq r|\nabla \Phi| \leq|x| \mathcal{A}(|x|)\|\varphi\|_{1, \mathcal{A} ; \Gamma_{\varrho / 4}^{2 \varrho}}, \quad \forall x \in G_{\varrho / 4}^{2 \varrho} .
$$

Similarly it follows from (4.13) as $\varrho \rightarrow+0$ that $f^{j}(0)=0$ for $j=1, \ldots, n$. Therefore we have for all $x \in G_{\varrho / 4}^{2 \varrho}$,

$$
\left|f^{j}(x)\right|=\left|f^{j}(x)-f^{j}(0)\right| \leq \mathcal{A}(r)\left[f^{j}\right]_{0, \mathcal{A} ; G_{\varrho / 4}^{2 \varrho}} .
$$

Consequently, estimating the right side of (4.26) and taking into account the inequalities obtained, we have

$$
\begin{aligned}
\sum_{i=1}^{n}\left|F^{i}\right|_{q ; G_{1 / 4}^{2}} \leq & c\left(q, G, \max _{j=1, \ldots, n}\left\{\sum_{i=1}^{n}\left\|a^{i j}\right\|_{0, \mathcal{A} ; G}, \sum_{i=1}^{n}\left\|a^{i}\right\|_{0, \mathcal{A} ; G}\right\}\right) \\
& \times \operatorname{mes} \Omega \cdot\left(\sum_{i=1}^{n}\left\|f^{i}\right\|_{0, \mathcal{A} ; G_{\varrho / 4}^{2 \varrho}}+\|\varphi\|_{1, \mathcal{A} ; \Gamma_{\varrho / 4}^{2 \varrho}}\right) .
\end{aligned}
$$

So all conditions of [5, Theorem 8.25$]$ are satisfied. By this theorem we get 


$$
\begin{aligned}
\sup _{x^{\prime} \in G_{1 / 2}^{1}} & \left|z\left(x^{\prime}\right)\right| \\
\leq & c\left(|z|_{2 ; G_{1 / 4}^{2}}+|G|_{n /(2(1-\alpha)) ; G_{1 / 4}^{2}}+\sum_{i=1}^{n} \mid F^{i} \mathbf{I}_{n /(1-\alpha) ; G_{1 / 4}^{2}}\right) \\
\leq & c\left(\left|\mathbf{|} z \mathbf{|}_{2 ; G_{1 / 4}^{2}}+\right| \mathbf{I} \mid \mathbf{|}_{n /(1-\alpha) ; G_{\varrho / 4}^{2 \varrho}}\right. \\
& \left.+\sum_{i=1}^{n}\left\|f^{i}\right\|_{0, \mathcal{A} ; G_{\varrho / 4}^{2 \varrho}}+\|\varphi\|_{1, \mathcal{A} ; \Gamma_{\varrho / 4}^{2 \varrho}}\right) .
\end{aligned}
$$

Setting $w(x)=u(x)-\varphi(x)$ we have for $w(x)$ the problem

$(\mathrm{DL})_{0,2 d}$

$$
\left\{\begin{array}{c}
\frac{\partial}{\partial x_{i}}\left(a^{i j}(x) w_{x_{j}}+a^{i}(x) w\right)+b^{i}(x) w_{x_{i}}+c(x) w \\
=G(x)+\frac{\partial F^{j}}{\partial x_{j}}, \quad x \in G_{0}^{2 d}, \\
w(x)=0, \quad x \in \Gamma_{0}^{2 d} \subset \partial G_{0}^{2 d},
\end{array}\right.
$$

where

$$
\begin{aligned}
G(x) & =g(x)-b^{i}(x) \Phi_{x_{i}}-c(x) \Phi(x), \\
F^{i}(x) & =f^{i}(x)-a^{i j}(x) \Phi_{x_{j}}-a^{i}(x) \Phi(x) .
\end{aligned}
$$

Moreover, by assumptions (i), (ii),

$$
\left|a^{i j}(x)-\delta_{i}^{j}\right| \leq\left\|a^{i j}\right\|_{0, \mathcal{A} ; G} \mathcal{A}(|x|), \quad x \in G .
$$

By [6, Theorem 1] there is a constant $c>0$ independent of $w, G, F^{i}$ such that

$$
\begin{aligned}
\int_{G_{0}^{\varrho}} r^{2-n}|\nabla w|^{2} d x \leq & c \varrho^{2 \lambda} \int_{G_{0}^{2 d}}\left\{|w(x)|^{2}+|\nabla w|^{2}+G^{2}(x)+\sum_{i=1}^{n}\left|F^{i}(x)\right|^{2}\right. \\
& +r^{4-n-2 \lambda} \mathcal{H}^{-1}(r) G^{2}(x)+r^{2-n-2 \lambda} \\
& \left.\times \mathcal{H}^{-1}(r) \sum_{i=1}^{n}\left|F^{i}(x)\right|^{2}\right\} d x, \quad \forall \varrho \in(0, d) .
\end{aligned}
$$

Our assumptions guarantee that the integral on the right side is finite. Since $z\left(x^{\prime}\right)=\varrho^{-1} \mathcal{A}^{-1}(\varrho) w\left(\varrho x^{\prime}\right)$ we obtain from (4.29),

$$
\begin{aligned}
\int_{G_{1 / 4}^{2}}\left|\nabla^{\prime} z\right|^{2} d x^{\prime} & \leq 2^{n-2} \varrho^{-2} \mathcal{A}^{-2}(\varrho) \int_{G_{\varrho / 4}^{2 \varrho}} r^{2-n}|\nabla w|^{2} d x \\
& \leq c \varrho^{2 \lambda-2} \mathcal{A}^{-2}(\varrho) \int_{G}\left\{|w|^{2}+|\nabla w|^{2}+G^{2}(x)\right.
\end{aligned}
$$




$$
\begin{aligned}
& +\sum_{i=1}^{n}\left|F^{i}(x)\right|^{2}+r^{4-n-2 \lambda} \mathcal{H}^{-1}(r) G^{2}(x) \\
& \left.+r^{2-n-2 \lambda} \mathcal{H}^{-1}(r) \sum_{i=1}^{n}\left|F^{i}(x)\right|^{2}\right\} d x .
\end{aligned}
$$

By assumptions (i)-(iv) we have

$$
\begin{aligned}
|G(x)|^{2} \leq & c\left\{|g|^{2}+\mathcal{A}^{2}(r)\left(r^{-2}|\nabla \Phi|^{2}+r^{-4} \Phi^{2}\right)\right\} \\
\sum_{i=1}^{n}\left|F^{i}(x)\right|^{2} \leq & c\left\{\sum_{i=1}^{n}\left|f^{i}(x)\right|^{2}\right. \\
& \left.+\max _{i, j=1, \ldots, n}\left(\left\|a^{i j}\right\|_{0, \mathcal{A} ; G},\left\|a^{i}\right\|_{0, \mathcal{A} ; G}\right)\left(|\nabla \Phi|^{2}+\Phi^{2}\right)\right\} .
\end{aligned}
$$

Applying now the Friedrichs inequality and taking into account (4.1), we obtain from (4.30), (4.31),

$$
\begin{aligned}
|z|_{2 ; G_{1 / 4}^{2} \leq}^{2} \leq & c_{0}\left|\nabla^{\prime} z\right|_{2 ; G_{1 / 4}^{2}}^{2} \\
\leq & c \varrho^{2 \lambda-2} \mathcal{A}^{-2}(\varrho) \int_{G}\left\{|w|^{2}+|\nabla w|^{2}+g^{2}(x)\right. \\
& +\sum_{i=1}^{n}\left|f^{i}(x)\right|^{2}+|\nabla \Phi|^{2}+\Phi^{2}+r^{4-n-2 \lambda} \mathcal{H}^{-1}(r) g^{2}(x) \\
& +r^{2-n-2 \lambda} \mathcal{H}^{-1}(r) \sum_{i=1}^{n}\left|f^{i}(x)\right|^{2} \\
& \left.+r^{2-n-2 \lambda} \mathcal{H}^{-1}(r)|\nabla \Phi|^{2}+r^{-2} \mathcal{A}^{2}(r)|\nabla \Phi|^{2}\right\} d x \\
\leq & \operatorname{const}\left\{|g|_{n /(1-\alpha) ; G}^{2}+\sum_{i=1}^{n}\left\|f^{i}\right\|_{0, \mathcal{A} ; G}^{2}+\|\varphi\|_{1, \mathcal{A} ; G}^{2}\right. \\
& +\int_{G}\left(|w|^{2}+|\nabla w|^{2}+r^{4-n-2 \lambda} \mathcal{H}^{-1}(r) g^{2}(x)\right. \\
& +r^{2-n-2 \lambda} \mathcal{H}^{-1}(r) \sum_{i=1}^{n}\left|f^{i}(x)\right|^{2} \\
& \left.\left.+r^{2-n-2 \lambda} \mathcal{H}^{-1}(r)|\nabla \Phi|^{2}\right) d x\right\}
\end{aligned}
$$

by assumptions (iii)-(v). By the definition of $z\left(x^{\prime}\right)$, inequalities (4.28), (4.32) and assumptions (i)-(v) we finally obtain

$$
|v|_{0 ; G_{1 / 4}^{2}} \leq|z|_{0 ; G_{1 / 4}^{2}}+\varrho^{-1} \mathcal{A}^{-1}(\varrho)|\varphi(\varrho \cdot)|_{0 ; G_{1 / 4}^{2}}
$$




$$
\begin{aligned}
\leq & c\left(\mid \mathbf{I} \mathbf{|}_{n /(1-\alpha) ; G}+\sum_{i=1}^{n}\left\|f^{i}\right\|_{0, \mathcal{A} ; G}+\|\varphi\|_{1, \mathcal{A} ; \partial G}\right. \\
& +\left\{\int _ { G } \left(|w|^{2}+|\nabla w|^{2}+r^{4-n-2 \lambda} \mathcal{H}^{-1}(r) g^{2}(x)\right.\right. \\
& +r^{2-n-2 \lambda} \mathcal{H}^{-1}(r) \sum_{i=1}^{n}\left|f^{i}(x)\right|^{2} \\
& \left.\left.\left.+r^{2-n-2 \lambda} \mathcal{H}^{-1}(r)|\nabla \Phi|^{2}\right) d x\right\}^{1 / 2}\right) .
\end{aligned}
$$

$3^{\circ}$. Returning to the variables $x, u(x)$, we now obtain from inequalities (4.16), (4.33),

$$
\begin{aligned}
\varrho^{-1} \mathcal{A}^{-1}(\varrho) \sup _{x \in G_{\varrho / 2}^{\varrho}}|u(x)| & +\mathcal{A}^{-1}(\varrho) \sup _{x \in G_{\varrho / 2}^{\varrho}}|\nabla u(x)| \\
& +\sup _{\substack{x, y \in G^{\varrho} \\
x \neq y}} \frac{|\nabla u(x)-\nabla u(y)|}{\mathcal{A}(\varrho) \mathcal{B}(|x-y|)} \\
\leq & c\left(\left.|| g\right|_{n /(1-\alpha) ; G}+\sum_{i=1}^{n}|| f^{i}\left\|_{0, \mathcal{A} ; G}+\right\| \varphi \|_{1, \mathcal{A} ; \partial G}\right. \\
& +\left\{\int _ { G } \left(|u|^{2}+|\nabla u|^{2}+r^{4-n-2 \lambda} \mathcal{H}^{-1}(r) g^{2}(x)\right.\right. \\
& +r^{2-n-2 \lambda} \mathcal{H}^{-1}(r) \sum_{i=1}^{n}\left|f^{i}(x)\right|^{2} \\
& \left.\left.\left.+r^{2-n-2 \lambda} \mathcal{H}^{-1}(r)|\nabla \Phi|^{2}\right) d x\right\}^{1 / 2}\right) .
\end{aligned}
$$

Setting $|x|=2 \varrho / 3$ we deduce from (4.34) the validity of (4.2), (4.3). This completes the proof of Theorem 3 .

Remark. As an example of $\mathcal{A}$ that satisfies all the conditions of Theorem 3, besides the function $r^{\alpha}$, one may take $\mathcal{A}(r)=r^{\alpha} \ln (1 / r)$, provided $\lambda \geq 1+\alpha$. In the case of $\mathcal{A}(r)=r^{\alpha}$ the result of [1] follows from Theorem 3 for a single equation and the estimate (4.2) coincides with [6, (10)].

\section{Global regularity and solvability}

Theorem 4. Let $\mathcal{A}$ be an $\alpha$-Dini function $(0<\alpha<1)$ that satisfies the conditions (1.5), (1.6), (4.1). Let $\bar{G} \backslash\{\mathcal{O}\}$ be a domain of class $C^{1, \mathcal{A}}$, and 
$\mathcal{O} \in \partial G$ be a conical point of $G$. Suppose that assumptions (i)-(iv) are valid and

$$
\int_{G}\left(c(x) \eta-a^{i}(x) D_{i} \eta\right) d x \leq 0, \quad \forall \eta \geq 0, \eta \in C_{0}^{1}(G) .
$$

Then the generalized problem (DL) has a unique solution $u \in C^{1, \mathcal{A}}(\bar{G})$ and we have the estimate

$$
\begin{aligned}
\|u\|_{1, \mathcal{A} ; G} \leq & c\left(\mid \mathbf{|} g \mathbf{|}_{n /(1-\alpha) ; G}+\sum_{i=1}^{n}\left\|f^{i}\right\|_{0, \mathcal{A} ; G}+\|\varphi\|_{1, \mathcal{A} ; \partial G}\right. \\
& +\left\{\int _ { G } \left(r^{4-n-2 \lambda} \mathcal{H}^{-1}(r) g^{2}(x)\right.\right. \\
& +r^{2-n-2 \lambda} \mathcal{H}^{-1}(r) \sum_{i=1}^{n}\left|f^{i}(x)\right|^{2} \\
& \left.\left.\left.+r^{2-n-2 \lambda} \mathcal{H}^{-1}(r)|\nabla \Phi|^{2}\right) d x\right\}^{1 / 2}\right) .
\end{aligned}
$$

Proof. The inequality (4.34) implies that

$$
\begin{aligned}
& |\nabla u(x)-\nabla u(y)| \\
& \leq c \mathcal{B}(|x-y|)\left(|g|_{n /(1-\alpha) ; G}+\sum_{i=1}^{n}\left\|f^{i}\right\|_{0, \mathcal{A} ; G}\right. \\
& \quad+\|\varphi\|_{1, \mathcal{A} ; \partial G}+\left\{\int _ { G } \left(|u|^{2}+|\nabla u|^{2}+r^{4-n-2 \lambda} \mathcal{H}^{-1}(r) g^{2}(x)\right.\right. \\
& \left.\left.\left.\quad+r^{2-n-2 \lambda} \mathcal{H}^{-1}(r) \sum_{i=1}^{n}\left|f^{i}(x)\right|^{2}+r^{2-n-2 \lambda} \mathcal{H}^{-1}(r)|\nabla \Phi|^{2}\right) d x\right\}^{1 / 2}\right)
\end{aligned}
$$

for all $x, y \in G_{\varrho / 2}^{\varrho}$ and all $\varrho \in(0, d)$.

From (4.34), (5.2) we now infer that $u \in C^{1, \mathcal{B}}\left(\overline{G_{0}^{d}}\right)$. Indeed, let $x, y \in \overline{G_{0}^{d}}$ and $\varrho \in(0, d)$. If $x, y \in G_{\varrho / 2}^{\varrho}$ then (5.2) holds. If $|x-y|>|\varrho|=|x|$ then by (4.34) we obtain

$$
\begin{aligned}
& \frac{|\nabla u(x)-\nabla u(y)|}{\mathcal{B}(|x-y|)} \\
& \quad \leq 2 c \mathcal{A}(|x|) \mathcal{B}^{-1}(|x|)\left(\left.\mathbf{I} g\right|_{n /(1-\alpha) ; G}+\|\varphi\|_{1, \mathcal{A} ; \partial G}\right. \\
& \quad+\sum_{i=1}^{n}\left\|f^{i}\right\|_{0, \mathcal{A} ; G}+\left\{\int _ { G } \left(|u|^{2}+|\nabla u|^{2}+r^{4-n-2 \lambda} \mathcal{H}^{-1}(r) g^{2}(x)\right.\right.
\end{aligned}
$$




$$
\begin{aligned}
& \left.\left.\left.+r^{2-n-2 \lambda} \mathcal{H}^{-1}(r) \sum_{i=1}^{n}\left|f^{i}(x)\right|^{2}+r^{2-n-2 \lambda} \mathcal{H}^{-1}(r)|\nabla \Phi|^{2}\right) d x\right\}^{1 / 2}\right) \\
\leq & 2 c \alpha\left(\mid \mathbf{I} g \mathbf{|}_{n /(1-\alpha) ; G}+\|\varphi\|_{1, \mathcal{A} ; \partial G}+\sum_{i=1}^{n}\left\|f^{i}\right\|_{0, \mathcal{A} ; G}\right. \\
& +\left\{\int _ { G } \left(|u|^{2}+|\nabla u|^{2}+r^{4-n-2 \lambda} \mathcal{H}^{-1}(r) g^{2}(x)\right.\right. \\
& \left.\left.\left.+r^{2-n-2 \lambda} \mathcal{H}^{-1}(r) \sum_{i=1}^{n}\left|f^{i}(x)\right|^{2}+r^{2-n-2 \lambda} \mathcal{H}^{-1}(r)|\nabla \Phi|^{2}\right) d x\right\}^{1 / 2}\right)
\end{aligned}
$$

in view of (1.3). Because of the condition (1.5) for the equivalence of $\mathcal{A}$ and $\mathcal{B}$, we derive $u \in C^{1, \mathcal{A}}\left(\overline{G_{0}^{d}}\right)$ and the estimate

$$
\begin{aligned}
\|u\|_{1, \mathcal{A} ; G_{0}^{d}} \leq & c\left(\mid \mathbf{I} \mathbf{|}_{n /(1-\alpha) ; G}+\sum_{i=1}^{n}\left\|f^{i}\right\|_{0, \mathcal{A} ; G}+\|\varphi\|_{1, \mathcal{A} ; \partial G}\right. \\
& +\left\{\int _ { G } \left(|u|^{2}+|\nabla u|^{2}+r^{4-n-2 \lambda} \mathcal{H}^{-1}(r) g^{2}(x)\right.\right. \\
& +r^{2-n-2 \lambda} \mathcal{H}^{-1}(r) \sum_{i=1}^{n}\left|f^{i}(x)\right|^{2} \\
& \left.\left.\left.+r^{2-n-2 \lambda} \mathcal{H}^{-1}(r)|\nabla \Phi|^{2}\right) d x\right\}^{1 / 2}\right)
\end{aligned}
$$

following from the above arguments.

By means of a partition of unity, from the bounds (3.1) of Theorem 2 and (5.3) we derive

$$
\begin{aligned}
\|u\|_{1, \mathcal{A} ; G} \leq & c\left(\mid \mathbf{g} g \mathbf{|}_{n /(1-\alpha) ; G}+\sum_{i=1}^{n}\left\|f^{i}\right\|_{0, \mathcal{A} ; G}+\|\varphi\|_{1, \mathcal{A} ; \partial G}\right. \\
& +|u|_{0 ; G}+\left\{\int _ { G } \left(|u|^{2}+|\nabla u|^{2}+r^{4-n-2 \lambda} \mathcal{H}^{-1}(r) g^{2}(x)\right.\right. \\
& +r^{2-n-2 \lambda} \mathcal{H}^{-1}(r) \sum_{i=1}^{n}\left|f^{i}(x)\right|^{2} \\
& \left.\left.\left.+r^{2-n-2 \lambda} \mathcal{H}^{-1}(r)|\nabla \Phi|^{2}\right) d x\right\}^{1 / 2}\right) .
\end{aligned}
$$

By the assumption (vi) that guarantees the uniqueness of the solution for the problem (DL), we have the bound [5, Corollary 8.7]

$$
\int_{G}\left(|u|^{2}+|\nabla u|^{2}\right) d x \leq C \int_{G}\left(g^{2}+\sum_{i=1}^{n}\left|f^{i}\right|^{2}+|\nabla \Phi|^{2}+\Phi^{2}\right) d x,
$$


which together with the global boundedness of weak solutions [5, Theorem 8.16], and the bound (5.4), leads to the desired estimate (5.1).

Finally, the global estimate (5.1) leads to the assertion on the unique solvability in $C^{1, \mathcal{A}}(\bar{G})$. This is proved by an approximation argument using the relevant propositions from [8] in the same way as in [5, Theorem 8.34].

Remark. The conclusion of Theorem 4 is best possible. This is shown for $\mathcal{A}(r)=r^{\alpha}, \lambda \geq 1+\alpha, \alpha \in(0,1)$, in [6] (see also examples in [2]).

\section{References}

[1] A. Azzam and V. Kondrat'ev, Schauder-type estimates of solutions of second order elliptic systems in divergence form in non-regular domains, Comm. Partial Differential Equations 16 (1991), 1857-1878.

[2] M. Borsuk, Best-possible estimates of solutions of the Dirichlet problem for linear elliptic nondivergence equations of second order in a neighbourhood of a conical point of the boundary, Math. USSR-Sb. 74 (1993), 185-201.

[3] C. Burch, The Dini condition and regularity of weak solutions of elliptic equations, J. Differential Equations 30 (1978), 308-323.

[4] S. Eŭdel'man and M. Matiǔchuk, The Cauchy problem for parabolic systems with coefficients having low smoothness, Ukrain. Mat. Zh. 22 (1970), 22-36 (in Russian).

[5] D. Gilbarg and N. Trudinger, Elliptic Partial Differential Equations of Second Order, Springer, Berlin, 1983.

[6] V. Kondrat'ev, I. Kopachek and O. Oleŭnik, On the best Hölder exponents for generalized solutions of the Dirichlet problem for a second-order elliptic equation, Mat. Sb. 131 (1986), 113-125 (in Russian); English transl.: Math. USSR-Sb. 59 (1988).

[7] G. Lieberman, The Dirichlet problem for quasilinear elliptic equations with continuously differentiable boundary data, Comm. Partial Differential Equations 11 (1986), $167-229$.

[8] E. Sperner, Schauder's existence theorem for $\alpha$-Dini continuous data, Ark. Mat. 19 (1981), 193-216.

Department of Applied Mathematics

Olsztyn University of Agriculture and Technology

10-957 Olsztyn-Kortowo, Poland

E-mail: borsuk@art.olsztyn.pl 